\section{Neue Erkenntnisse zum Mechanismus des Lungenkrebs}

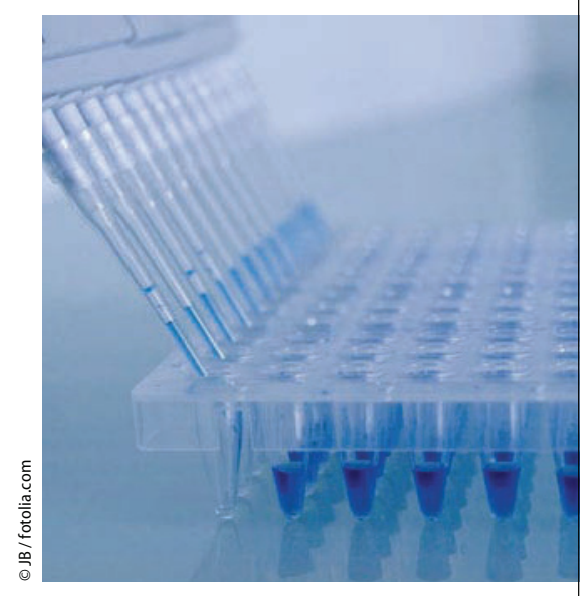

In einer neuen Studie klärten Forscher aus den USA und Italien den Mechanismus auf, der die Rb2/p130-Genexpression in Lungenfibroblasten kontrolliert. Außerdem konnten sie die Folgen einer Fehlregulation des Gens auf die proliferativen Eigenschaften der Zellen charakterisieren.

Am Kontrollmechanismus der Rb2/ p130-Genexpression in Lungenfibroblasten sind die Proteine CCCTC-Bindungsfaktor (CTCF) und BORIS beteiligt. CTCF bindet an die Promotorregion des $\mathrm{Rb} 2 / \mathrm{p} 130$-Gens und beeinflusst so dessen Transkription. In den Krebszellen ist die Funktion von CTCF durch das Protein BORIS beeinträchtigt und somit auch dessen Kontrolle über die Transkription des Rb2/p130-Gens. In der Studie konnte gezeigt werden, dass Rb2/p130 in kleinzelligen Bronchialkarzinomzellen nur gering exprimiert ist, wohingegen es in nichtkleinzelligen Krebszellen überexprimiert wird.

Die Ergebnisse dieser Studie liefern wichtige Informationen um die proliferativen und antiproliferativen Signale $z u$ verstehen, die von $\mathrm{Rb} 2 / \mathrm{p} 130$ ausgelöst werden.

Literatur: Fiorentino FP, Macaluso M, Miranda F et al (2011) CTCF and BORIS regulate Rb2/p130 gene transcription: a novel mechanism and a new paradigm for understanding the biology of lung cancer. Mol Cancer Res 9(2):225-233

\section{»Die Methode der Wahl zur \\ Darstellung von Oligometastasen ist die PET/CT-Bildgebung «}

\section{Was ist eine Oligometastasierung?}

Hellmann und Weichselbaum bezeichnen die Oligometastasierung als eine eingegrenzte, lokoregionale Tumorbelastung [3]. Unter Oligometastase oder solitärer Metastase versteht man somit einen biologisch weniger aggressiven Status, in welchem die Anzahl (solitärer Herd), die Größe $(<5 \mathrm{~cm})$ und die Lokalisation (limitiert an einem Organ) der metastatischen Foci über eine gewisse Zeit konstant geblieben sind [1]. Heute wird dieser Begriff für alle isolierten Fernmetastasen verwendet.

Aus dieser Definition ergibt sich auch die potenziell kurative Behandlung dieser Krankheitsform.

\section{Welche Organe sind am meisten betroffen?}

Solitäre Metastasen eines NSCLC finden sich bei ca. 7\% der Patienten. Meistens handelt es sich um synchrone oder metachrone Metastasen in verschiedenen Lungenlappen. Extrapulmonal treten die Oligometastasen bevorzugt in Gehirn, Knochen, Leber und Nebennieren auf [2].

\section{Staging}

Das Thema Staging bei Patienten mit Lungenkarzinom ist komplex. In den interdisziplinären S3-Leitlinien zur Prävention, Diagnostik, Therapie und Nachsorge des Lungenkarzinoms hat sich die PET/CT-Bildgebung als eine bedeutende Komponente beim Staging und bei der Suche nach Fernmetastasen des Lungenkarzinoms herauskristallisiert [4].

$\mathrm{Zu}$ Zeiten der dezidierten PET-Bildgebung galt die Beurteilung des Primärtumors als alleinige Domäne der CT. Mit zunehmender Verbreitung der PET/CT-Hybridgeräte ist auf Basis des gesichert übereinstimmenden Ortskoordinatensystems ein diagnostischer Zugewinn im Vergleich zur separat akquirierten PET- bzw. CTBildgebung möglich [4][5]. Hauptvorteil der FDG-PET/CT ist, dass mit nur einem
Bildgebungsverfahren gleichzeitig Metastasen in Lunge, Lymphknoten, Knochen und Weichteilstrukturen nachgewiesen werden können. Insgesamt kann die kombinierte FDG-PET/CT mit einem sowohl positiven als auch negativen prädiktiven Wert von $>95 \%$ für alle Lokalisationen zwischen Schädelbasis und Leiste aktuell als Referenzmethode zum nichtinvasiven Staging von Lungenkarzinomen betrachtet werden. Sie ist somit auch die Methode der Wahl zur Darstellung der Oligometastasen.

Aufgrund des physiologisch hohen Hintergrunds ist der Stellenwert der FDG-PET im Gehirn jedoch limitiert. In einer Vergleichsstudie wurde die Sensitivität der FDG-PET hier mit 24\%, die der MRT mit $88 \%$ angegeben. Für eine suffiziente Ganzkörperdiagnostik sollten diese beiden Verfahren daher miteinander kombiniert werden ([4], • Abb. 1).

\section{Diagnose und Therapie}

\section{Lungenmetastasen}

Eine durch mehrere internationale Fachorganisationen durchgeführte extensive Aufarbeitung der Langzeitergebnisse des behandelten Lungenkarzinoms führte $2007 \mathrm{zu}$ einer Revision der TNM-Klassifikation. In der neuen 7. Edition der TNM-Klassifikation wurden intrapulmonale Metastasen im selben Lungenlobus als $\mathrm{T}_{3}$, in demselben Lappen als $\mathrm{T}_{4}$ und in der kontralateralen Lunge als Mia eingestuft [6].

Kontroverse Meinungen bestehen bezüglich der Differenzialdiagnose zwischen einem sekundären Lungenkarzinom und Metastasen eines bekannten primären Lungenkarzinoms. Für die klinische Differenzierung dieser beiden Entitäten haben Martini und Melamet schon 1975 Kriterien aufgelistet [7]. In der letzten Dekade haben sich neue histologische sowie molekulargenetische Instrumente entwickelt, die eine Differenzialdiagnose ermöglichen. Mit Hilfe dieser Techniken sollte die Frage, ob ähnliche Mutationen wie bei einem Primärtumor vorliegen, beantwortet werden. Somit könnte man 